\title{
Prognostic Impact of Resection Margin Status in Distal Pancreatectomy for Ductal Adenocarcinoma
}

\author{
Mushegh A. Sahakyan, MD, $\mathrm{PhD}^{1,2,3}$ (1), Caroline S. Verbeke, MD, $\mathrm{PhD}^{4,5}$, Tore Tholfsen, $\mathrm{MD}^{6}$, \\ Dejan Ignjatovic, $\mathrm{MD}, \mathrm{PhD}^{4,7}$, Dyre Kleive, $\mathrm{MD}, \mathrm{PhD}^{6}$, Trond Buanes, $\mathrm{MD}, \mathrm{PhD}^{4}$, Kristoffer Lassen, $\mathrm{MD}, \mathrm{PhD}^{6,8}$, \\ Bård I. Røsok, $\mathrm{MD}, \mathrm{PhD}^{6}$, Knut Jørgen Labori, $\mathrm{MD}, \mathrm{PhD}^{4,6}$, and Bjørn Edwin, $\mathrm{MD}, \mathrm{PhD}^{1,4,6}$ \\ ${ }^{1}$ The Intervention Centre, Rikshospitalet, Oslo University Hospital, Oslo, Norway; ${ }^{2}$ Division of Emergencies and Critical \\ Care, Department of Research and Development, Oslo University Hospital, Oslo, Norway; ${ }^{3}$ Department of Surgery N1, \\ Yerevan State Medical University, Yerevan, Armenia; ${ }^{4}$ Institute of Clinical Medicine, University of Oslo, Oslo, Norway; \\ ${ }^{5}$ Department of Pathology, Rikshospitalet, Oslo University Hospital, Oslo, Norway; ${ }^{6}$ Department of Hepato-Pancreato- \\ Biliary Surgery, Rikshospitalet, Oslo University Hospital, Oslo, Norway; ${ }^{7}$ Department of Digestive Surgery, Akershus \\ University Hospital, Lørenskog, Norway; ${ }^{8}$ Institute of Clinical Medicine, University of Troms $\varnothing$, Troms $\emptyset$, Norway
}

\begin{abstract}
Background. Resection margin status is considered one of the few surgeon-controlled parameters affecting prognosis in pancreatic ductal adenocarcinoma (PDAC). While studies mostly focus on resection margins in pancreatoduodenectomy, little is known about their role in distal pancreatectomy (DP). This study aimed to investigate resection margins in DP for PDAC.

Methods. Patients who underwent DP for PDAC between October 2004 and February 2020 were included $(n=124)$. Resection margins and associated parameters were studied in two consecutive time periods during which different pathology examination protocols were used: non-standardized (period 1: 2004-2014) and standardized (period 2: 2015-2020). Microscopic margin involvement $(R 1)$ was defined as $\leq 1 \mathrm{~mm}$ clearance.
\end{abstract}

Caroline S. Verbeke and Mushegh A. Sahakyan share first authorship on this work.

Supplementary Information The online version contains supplementary material available at https://doi.org/10.1245/s10434021-10464-6.

(C) The Author(s) 2021

First Received: 24 March 2021

Accepted: 29 June 2021;

Published Online: 22 July 2021

M. A. Sahakyan, MD, $\mathrm{PhD}$

e-mail: sahakyan.mushegh@gmail.com
Results. Laparoscopic and open resections were performed in 117 (94.4\%) and 7 (5.6\%) patients, respectively. The $R 1$ rate for the entire cohort was $73.4 \%$, increasing from $60.4 \%$ in period 1 to $83.1 \%$ in period $2(p=0.005)$. A significantly higher $R 1$ rate was observed for the posterior margin $(35.8$ vs. $70.4 \%, p<0.001)$ and anterior pancreatic surface (based on a $0 \mathrm{~mm}$ clearance; 18.9 vs. $35.4 \%, p=0.045$ ). Pathology examination period, poorly differentiated PDAC, and vascular invasion were associated with $R 1$ in the multivariable model. Extended DP, positive anterior pancreatic surface, lymph node ratio, perineural invasion, and adjuvant chemotherapy, but not $R 1$, were significant prognostic factors for overall survival in the entire cohort.

Conclusions. Pathology examination is a key determinant of resection margin status following DP for PDAC. A high $R 1$ rate is to be expected when pathology examination is meticulous and standardized. Involvement of the anterior pancreatic surface affects prognosis.

Surgery remains the cornerstone of treatment for pancreatic cancer. ${ }^{1}$ Resection margin status is considered one of the few surgeon-controlled parameters affecting prognosis. ${ }^{2-4}$ Hence, surgeons strive to improve the quality of surgery to avoid positive resection margins $(R 1)$. At the same time, the reported $R 1$ rate for pancreatectomy lies between $17 \%$ and $85 \% .^{5}$ On the one hand, the wide variation is attributed to different definitions used for $R 1,{ }^{5,6}$ and, on the other hand, recent studies suggest that the 
meticulousness of the pathology work-up significantly influences the incidence of $R 1 .^{7-9}$ Therefore, the $R 1$ rate can also be regarded as a quality marker for pathology.

Multiple studies have addressed the issue of resection margins in pancreatic cancer surgery, ${ }^{10-13}$ and while these studies focus on pancreatoduodenectomy, little is known about resection margin status and its clinical relevance in the case of distal pancreatectomy (DP). The latter is a less common procedure for pancreatic cancer compared with pancreatoduodenectomy. Furthermore, resection margins in DP are not the same given the differences in anatomy and resected structures in both specimen types. Thus, examination of the resection margins in DP requires, to some extent, a different approach. Most importantly, while pathology examination protocols for DP specimens have been published, ${ }^{14}$ there is currently no international consensus.

This study examines resection margin status in DP specimens with ductal adenocarcinoma. Pathology examination methods, factors associated with $R 1$, and the impact of margin status on patient prognosis are investigated.

\section{METHODS}

Patients operated for pancreatic ductal adenocarcinoma (PDAC) in the body or tail of the pancreas at Oslo University Hospital, Rikshospitalet, between October 2004 and February 2020, were included in this study. All patients were evaluated by the multidisciplinary team before being referred to surgery, and patients without preoperative radiological evidence of tumor invasion into adjacent major vessels were referred to laparoscopic DP. Tumor invasion into adjacent organs necessitating an extended DP was not considered a contraindication for a laparoscopic approach. Patients with preoperative radiological suspicion of tumor invasion into major vessels were referred to open surgery.

Neoadjuvant chemotherapy was not administered to patients with primary resectable PDAC. Those with borderline resectable/locally advanced PDAC were treated with preoperative chemotherapy (preferably FOLFIRINOX). Patients who eventually underwent DP following preoperative chemotherapy were included in this study. In highly selected patients in whom a single distant metastasis or limited peritoneal spread was detected intraoperatively, synchronous metastasectomy was performed. Surgical technique, adjuvant therapy, and follow-up strategy have been described elsewhere. ${ }^{15-17}$
Study Design and Exclusion Criteria

Data on patient demographics, clinical characteristics, perioperative outcomes, and pathology findings were retrieved from a prospectively maintained database. Resection margin status and other pathology parameters were studied in two consecutive time periods during which different approaches to pathology examination were used: non-standardized (period 1: 2004-2014) and standardized (period 2: 2015-2020). Factors associated with an $R 1$ resection were investigated. Patients with incomplete data $(n=14)$ and those participating in ongoing randomized controlled trials $(n=10)$ were excluded from the analysis, as were patients with tumors other than PDAC or its subtypes, ${ }^{18}$ as well as those with adenocarcinoma associated with intraductal papillary mucinous neoplasia.

The impact of resection margin status on long-term oncologic outcomes (recurrence and survival) was evaluated in patients with non-metastatic PDAC. Those with limited, intraoperatively detected metastatic disease were excluded from these analyses. Survival data were obtained from the Norwegian National Population Registry, and local hospitals were contacted, when necessary. The study was approved by the Hospital Review Board according to the guidelines provided by the Regional Ethics Committee. For patients not included in the Thematic Pancreatic Tumour Project (REK ref. 2015/738), the Regional Ethics Committee waived written consent (REK Sør-Øst B 2018/1060) for those patients who had died at date of last follow-up.

This study was performed according to the Strengthening the Reporting of Observational Studies in Epidemiology (STROBE) guidelines. ${ }^{19}$ Comprehensive information on the distribution of patients' race or ethnicity was not available due to the retrospective nature of this study.

\section{Specimen Grossing}

During period 1, grossing of DP specimens was performed by trained technical laboratory staff. Following fixation and color-coded inking of the anterior and posterior specimen surfaces, the specimen was sliced either along the longitudinal axis of the pancreatic body/tail, in the sagittal plane, or a combination of both, as described previously. ${ }^{20}$ Samples were taken from the tumor-bearing pancreas and from palpated peripancreatic or hilar lymph nodes. In the case of extended DP specimens, dissection and tissue sampling was left to the discretion of the laboratory staff and supervising consultant pathologist.

During period 2, specimen grossing was performed by a specialist consultant pathologist (CSV) following a procedure that has been described previously. ${ }^{21,22}$ The anterior 
and posterior surfaces of the pancreas, as well as the transection margins of the splenic artery and vein, were inked in different colors. The specimen was thinly sliced (3 mm slice thickness) in the sagittal plane. Numerous tissue blocks were taken from the grossly visible tumor onto the specimen surfaces. All visible lymph nodes were completely embedded, and peripancreatic or hilar adipose tissue that was not clearly devoid of lymph nodes was extensively sampled. In the case of neoadjuvant treatment, all tissues and structures that were not entirely normal were embedded. The same dissection and sampling procedures were followed for extended resection specimens, with extra tissue samples being taken from the tumor onto the additionally resected structures and their relevant surfaces, in particular circumferential resection margins (e.g., the surface of the soft tissue plane between the tumor and left adrenal).

\section{Definitions}

Standard and extended DP, as well as concomitant (noncontiguous organ) resections were defined according to the consensus criteria suggested by the International Study Group for Pancreatic Surgery (ISGPS). ${ }^{23}$ The Accordion Severity Grading System was applied to classify postoperative complications; ${ }^{24}$ grade III or higher complications were considered severe. Postoperative pancreatic fistula (POPF) and hemorrhage were defined and graded according to the ISGPS. ${ }^{25,26}$

Tumor size was defined as the largest diameter reported on pathology, and $R 1$ was defined as a $1 \mathrm{~mm}$ or less clearance to a margin and $0 \mathrm{~mm}$ clearance to the anterior pancreatic surface. Lymph node ratio was calculated by dividing the number of positive lymph nodes by the total number of examined lymph nodes. TNM stage was determined based on the 8th edition of the American Joint Committee on Cancer/Union for International Cancer Control (AJCC/UICC) staging system for pancreatic cancer. ${ }^{27,28}$

Recurrence was defined as radiological evidence of intra-abdominal soft tissue around the surgical site and/or distant metastases, and was classified as local recurrence, distant (hematogenous) metastasis, or peritoneal carcinomatosis. Patients without recurrence were censored at the last follow-up. Overall survival was estimated from the date of surgery until the date of death or the date of censoring (1 December 2020).

\section{Statistics}

Continuous data were expressed as median (range) or mean ( \pm standard deviation), while frequencies (percentages) were applied for categorical data. The two-sample
Student's $t$-test and Mann-Whitney $U$ test were used for normally and non-normally distributed continuous variables, respectively, and the Chi-square and Fisher's exact tests were applied for the frequencies. A two-tailed $p$-value $<0.05$ was considered statistically significant. A multivariable binary logistic regression model was used to examine the association between $R 1$ and clinicopathological parameters that were significant in the univariable analysis.

The Kaplan-Meier method was used to estimate survival and to plot survival curves, with survival being described as median (95\% confidence interval). Univariable and multivariable Cox regression analyses were applied to identify prognostic factors for survival. Parameters significant at $p<0.1$ in the univariable analysis were added to the multivariable model with backward selection. A two-tailed $p$-value $<0.05$ was considered statistically significant.

\section{RESULTS}

\section{Perioperative Results}

Overall, 124 patients underwent DP for PDAC within the study period. Preoperative chemotherapy was administered to $9(7.3 \%)$ patients. Patient characteristics and perioperative data are presented in electronic supplementary Table 1. Standard resections were performed in 68.5\% of cases, and laparoscopic DP was initiated in 117 (94.4\%) patients, 9 (7.7\%) of whom underwent conversion. Fiftytwo (41.9\%) patients had complications, including 30 (24.2\%) patients with severe complications. Ninety-day mortality was $0.8 \%(1 / 124)$ and median length of stay was 5 days (3-49).

\section{Pathology Findings and Predictors for RI}

Most patients (54\%) had pT3 cancer without a significant difference between both periods. The proportion of node-negative ( $\mathrm{pN} 0$ ) specimens decreased from $43.3 \%$ in period 1 to $19.7 \%$ in period 2 (Table 1), while the incidence of pN2 significantly increased (13.2 vs. 39.4\%, $p<0.001)$. The lymph node yield also significantly increased, from 8 in period 1 to 20 in period $2(p<0.001)$. During period 2, a significantly larger number of tissue blocks from the tumor and adjacent structures and margins were examined ( 14 vs. $9, p<0.001)$.

The $R 1$ rate for the entire cohort was $73.4 \%$, increasing from $60.4 \%$ in period 1 to $83.1 \%$ in period $2(p=0.005)$. Furthermore, the $R 1$ rate at the posterior margin increased from $35.8 \%$ to $70.4 \%(p<0.001)$, and, for the anterior pancreatic surface, the $R 1$ rate increased from $18.9 \%$ to 
TABLE $1 \mathrm{R}$ status and other pathology-based tumor features in distal pancreatectomy specimens

\begin{tabular}{|c|c|c|c|c|}
\hline Parameter & Total $[n=124]$ & Period $1[n=53]$ & Period $2[n=71]$ & $p$ value \\
\hline Tumor size, mm [mean (SD)] & $43.6(18.4)$ & $40.9(17.6)$ & $45.5(18.8)$ & 0.17 \\
\hline pT stage & & & & 0.79 \\
\hline pT1 & $12(9.7)$ & $6(11.3)$ & $6(8.5)$ & \\
\hline pT2 & $45(36.3)$ & $20(37.7)$ & $25(35.2)$ & \\
\hline pT3 & $67(54)$ & $27(50.9)$ & $40(56.3)$ & \\
\hline pN stage & & & & $<0.001$ \\
\hline pNO & $37(29.8)$ & $23(43.4)$ & $14(19.7)$ & \\
\hline $\mathrm{pN} 1$ & $52(41.9)$ & $23(43.4)$ & $29(40.8)$ & \\
\hline $\mathrm{pN} 2$ & $35(28.2)$ & $7(13.2)$ & $28(39.4)$ & \\
\hline M1 stage & $11(8.9)$ & $5(9.4)$ & $6(8.5)$ & 1.0 \\
\hline Lymph node yield [median (range)] & $16(1-48)$ & $8(1-48)$ & $20(6-41)$ & $<0.001$ \\
\hline Poor differentiation ${ }^{\mathrm{a}}$ & $38(33)$ & $11(22)$ & $27(41.5)$ & 0.027 \\
\hline Vascular invasion & $88(71)$ & $35(66)$ & $53(74.6)$ & 0.29 \\
\hline Lymphatic invasion & $84(67.7)$ & $29(54.7)$ & $55(77.5)$ & 0.007 \\
\hline Perineural invasion & $110(88.7)$ & $47(88.7)$ & $63(88.7)$ & 1.0 \\
\hline Tumor blocks examined [median (range)] & $12(4-27)$ & $9(4-22)$ & $14(6-27)$ & $<0.001$ \\
\hline$R$ status & & & & 0.005 \\
\hline$R 0$ & $33(26.6)$ & $21(39.6)$ & $12(16.9)$ & \\
\hline$R 1$ & $91(73.4)$ & $32(60.4)$ & $59(83.1)$ & \\
\hline \multicolumn{5}{|l|}{ Positive margins and surfaces } \\
\hline Transection pancreas & $16(12.9)$ & $8(15.1)$ & $8(11.3)$ & 0.53 \\
\hline Posterior & $69(55.6)$ & $19(35.8)$ & $50(70.4)$ & $<0.001$ \\
\hline Anterior surface & $35(28.2)$ & $10(18.9)$ & $25(35.2)$ & 0.045 \\
\hline Transection splenic artery/vein & $7(5.6)$ & $-^{\mathrm{b}}$ & $7(9.9)$ & - \\
\hline$>1$ positive margin/surface & $34(27.4)$ & $6(11.3)$ & $28(39.4)$ & 0.001 \\
\hline
\end{tabular}

Data are expressed as $n(\%)$ unless otherwise specified

$S D$ standard deviation

${ }^{a}$ Not applicable to tumors treated with preoperative chemotherapy

${ }^{\mathrm{b}}$ Not studied in period 1

$35.4 \%(p=0.045)$. In addition, involvement of more than one margin or surface also increased significantly (11.3 vs. $39.4 \%, p=0.001)$. The pathology examination period, grade of differentiation, tumor size, $\mathrm{pT}$ and $\mathrm{pN}$ stages, and lymphatic and vascular invasion correlated with $R 1$ in the univariable model (Table 2). In multivariable analysis, pathology examination period, poor differentiation, and presence of vascular invasion were independent predictors for $R 1$.

\section{Long-Term Oncologic Outcomes}

After excluding patients with distant metastasis $(n=11), 113$ patients with PDAC were analyzed for longterm oncologic outcomes, of whom 34 (30.1\%) underwent extended DP and 79 (69.9\%) had standard DP (23 R0 and $56 R 1)$. $\mathrm{R}$ status and positive resection margins/surfaces in extended DP specimens are presented in electronic supplementary Table 2.

Median follow-up was 19 months (3-108). Patients were comparable in terms of the administration of preoperative and adjuvant chemotherapy (Table 3). Recurrence was observed in $86(76.1 \%)$ patients. Extended DP resulted in a significantly higher incidence of recurrence compared with $R 0$ standard and $R 1$ standard DP (91.2 vs. 69.6 vs. $69.6 \%, p=0.048)$. No statistically significant association was found between the recurrence site and $\mathrm{R}$ status/extent of surgery. The site of resection margin/surface involvement did not correlate with either the development of recurrence or the site of recurrence (electronic supplementary Table 3).

Overall median survival was 20 months (15.2-24.8). Extended DP was associated with a significantly shorter median survival compared with standard DP $(R 1$ and $R 0)$, 
TABLE 2 Clinical and pathology parameters associated with $R 1$ status following distal pancreatectomy for adenocarcinoma: univariable and multivariable analysis

\begin{tabular}{|c|c|c|c|c|c|}
\hline \multirow[t]{2}{*}{ Parameter } & \multicolumn{3}{|c|}{ Univariable analysis } & \multicolumn{2}{|c|}{ Multivariable analysis } \\
\hline & $R 0[n=33]$ & $R 1[n=91]$ & $p$-Value & OR $(95 \% \mathrm{CI})$ & $p$-Value \\
\hline Age, years [mean $(\mathrm{SD})]$ & $67.7(9.8)$ & $66.7(9.9)$ & 0.6 & & \\
\hline Body mass index [mean (SD)] & $23.8(4.2)$ & $25.5(4.6)$ & 0.07 & & \\
\hline Male sex & $19(57.6)$ & $53(58.2)$ & 0.95 & & \\
\hline Preoperative chemotherapy & $2(6.1)$ & $7(7.7)$ & 1.0 & & \\
\hline Surgeon (senior consultant) & $27(81.8)$ & $71(78)$ & 0.65 & & \\
\hline Standard resection & $27(81.8)$ & $58(63.7)$ & 0.06 & & \\
\hline Laparoscopic procedure & $33(100)$ & $84(92.3)$ & 0.19 & & \\
\hline Conversion $^{\mathrm{a}}$ & $1(3)$ & $8(9.5)$ & 0.44 & & \\
\hline Standardized pathology work-up & $12(36.4)$ & $59(64.8)$ & 0.005 & $2.9(1.14-7.2)$ & 0.025 \\
\hline Poor differentiation ${ }^{\mathrm{b}}$ & $4(12.9)$ & $34(40.5)$ & 0.006 & $3.4(1.03-11.4)$ & 0.045 \\
\hline Tumor size, mm [mean (SD)] & $36.8(18.2)$ & $46(18)$ & 0.014 & - & NS \\
\hline pT stage & & & 0.019 & & \\
\hline pT1 & $7(21.2)$ & $5(5.5)$ & & Reference & - \\
\hline $\mathrm{pT} 2$ & $13(39.4)$ & $32(35.2)$ & & - & NS \\
\hline pT3 & $13(39.4)$ & $54(59.3)$ & & - & NS \\
\hline pN stage & & & $<0.01$ & & \\
\hline NO & $18(54.5)$ & $19(20.9)$ & & Reference & - \\
\hline$N 1$ & $12(36.4)$ & $40(44)$ & & - & NS \\
\hline$N 2$ & $3(9.1)$ & $32(35.2)$ & & - & NS \\
\hline Splenic vein invasion ${ }^{c}$ & 4 (28.6) & $34(56.7)$ & 0.06 & & \\
\hline Splenic artery invasion $^{c}$ & $0(0)$ & $5(8.3)$ & 0.58 & & \\
\hline Lymphatic invasion & $17(51.5)$ & $67(73.6)$ & 0.02 & - & NS \\
\hline Vascular invasion & $15(45.5)$ & $73(80.2)$ & $<0.01$ & $3.65(1.44-9.3)$ & 0.006 \\
\hline Perineural invasion & $28(84.8)$ & $82(90.1)$ & 0.52 & & \\
\hline
\end{tabular}

Data are expressed as $n(\%)$ unless otherwise specified

$O R$ odds ratio, $C I$ confidence interval, $N S$ non-significant, $S D$ standard deviation

${ }^{\mathrm{a}}$ Calculated for laparoscopic cases

${ }^{\mathrm{b}}$ Not applicable in patients treated with preoperative chemotherapy

${ }^{\mathrm{c}}$ Invasion into the splenic vein/artery was not reported in period 1 ; c-statistic $=0.84$

TABLE 3 Chemotherapy and disease recurrence in patients with non-metastatic ductal adenocarcinoma undergoing standard $(R 0 / R 1)$ and extended distal pancreatectomy

\begin{tabular}{|c|c|c|c|c|}
\hline Parameter & Standard $R 0[n=23]$ & Standard $R 1[n=56]$ & Extended $[n=34]$ & $p$-Value \\
\hline Preoperative chemotherapy & $0(0)$ & $5(8.9)$ & $1(2.9)$ & 0.33 \\
\hline Adjuvant chemotherapy & $18(78.3)$ & $35(62.5)$ & $22(64.7)$ & 0.39 \\
\hline Recurrence & $16(69.6)$ & $39(69.6)$ & $31(91.2)$ & 0.048 \\
\hline Local & $6(26.1)$ & $18(32.1)$ & $11(32.4)$ & 0.85 \\
\hline Distant metastases & $11(47.8)$ & $24(42.9)$ & $19(55.9)$ & 0.49 \\
\hline Peritoneal carcinomatosis & $2(8.7)$ & $7(12.5)$ & $10(29.4)$ & 0.08 \\
\hline
\end{tabular}

Data are expressed as $\mathrm{n}(\%)$

i.e. 14 vs. 20 vs. 43.2 months $(p=0.003)$ (Fig. 1$)$. Univariable analysis revealed that extended resection, perioperative red blood cell transfusion, $\mathrm{pN}$ stage, lymph node ratio, resection margin status, anterior pancreatic surface, number of resection margins/surfaces involved, vascular and perineural invasion, and adjuvant chemotherapy were associated with survival (Table 4). In the multivariable model, extended resection, anterior 
FIG. 1 Survival Following standard $(R 0 / R 1)$ and extended distal pancreatectomy in patients with non-metastatic ductal adenocarcinoma. Cum cumulative, $C I$ confidence interval

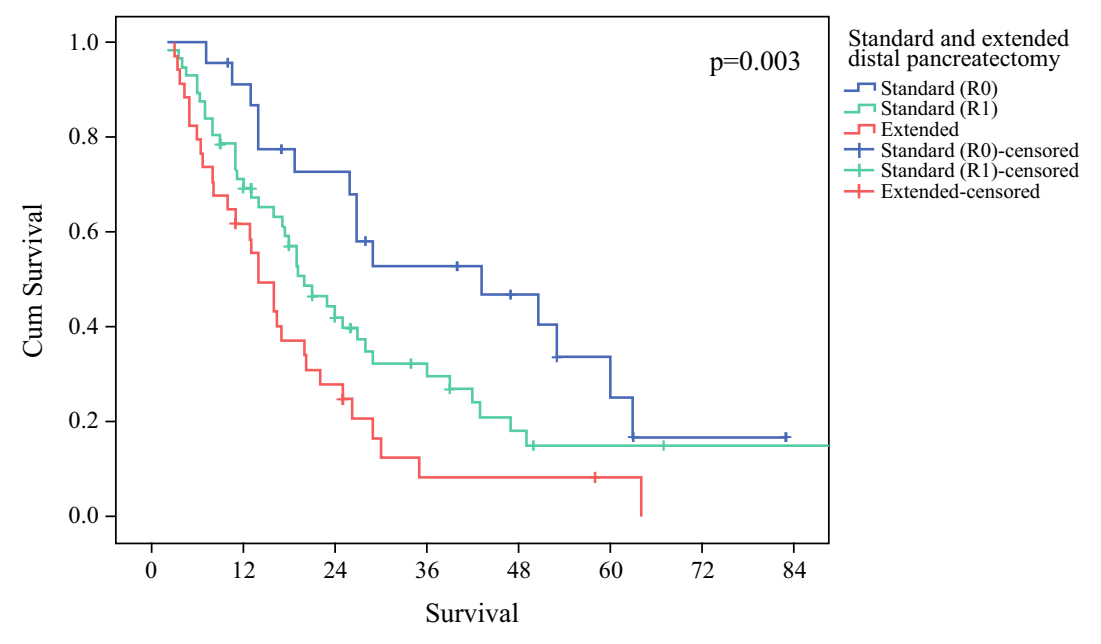

\begin{tabular}{l|cccc|ccc}
\hline \multirow{2}{*}{ Parameter } & \multicolumn{3}{|c|}{ Survival } & \multicolumn{3}{c}{ p-value } \\
\cline { 2 - 5 } & Median (95\% CI) & 1-year & 3-year & 5 -year & & 0.06 & 0.001 \\
\hline Standard R0 & $43.2(14-72.4)$ & $91.1 \%$ & $52.8 \%$ & $25.1 \%$ & & 0.04 \\
Standard R1 & $20(14.6-25.4)$ & $69.2 \%$ & $29.6 \%$ & $14.9 \%$ & 0.06 & & 0.04 \\
Extended & $14(9.9-18.1)$ & $61.8 \%$ & $8.2 \%$ & $8.2 \%$ & 0.001 & 0.04
\end{tabular}

pancreatic surface, lymph node ratio, perineural invasion, and adjuvant chemotherapy, but not overall resection margin status, were predictors for survival. Prognostic factors in different study periods are presented in electronic supplementary Table $4 . R 1$ was statistically significant in the univariate analysis of period 2 , but not in the multivariable analysis.

\section{DISCUSSION}

In this series from a high-volume tertiary referral center of pancreatic surgery, the rate of microscopic margin involvement following DP for PDAC was $73.4 \%$ during the entire study period (2004-2020). However, following the introduction of a detailed standardized pathology examination procedure for specimen grossing in 2015, the $R 1$ rate increased significantly, from $60.4 \%$ to $83.1 \%$. Concomitantly, the incidence of microscopic involvement of the posterior resection margin and the anterior pancreatic surface increased significantly. Given the differences observed after the implementation of the standardized pathology work-up, we cautiously assume that the exact $R 1$ rate during the entire study period was higher than $73.4 \%$. The accuracy of the standardized specimen examination also had an impact on the lymph node yield and nodal status, as the number of detected lymph nodes and the rate of positive lymph nodes were significantly higher in period 2, which is in line with a previous report from our group. ${ }^{20}$
An important question to address is whether the $R 1$ rate solely relates to the extent of surgery or whether it also reflects the quality of the pathology examination. In our center, neither the surgical technique nor the indications for DP in PDAC changed during the study period. Furthermore, the workload was similar and the same consultant surgeons were involved in both study periods. Therefore, the significant increase in the $R 1$ rate observed in this study is likely to be attributed to the implementation of a meticulous, standardized pathology work-up rather than to any aspect of surgery. This is also confirmed in the multivariable analysis demonstrating that the pathology examination period is a significant factor associated with $R 1$.

Previous series on DP for PDAC have reported $R 1$ rates that are considerably lower and vary significantly, from $0 \%$ to $59 \% .^{29-33}$ At the same time, data provided in these studies are quite heterogenous. A probable explanation for the wide variation is the lack of consensus as to which specimen surfaces are to be included in the assessment of the margin status. Furthermore, different definitions for $R 1$ have been used $(0 \mathrm{~mm}$ or $\leq 1 \mathrm{~mm}$ clearance). Recent studies based on the definitions of $R 1$ according to the Royal College of Pathologists $(\leq 1 \mathrm{~mm}$ clearance; $0 \mathrm{~mm}$ clearance at the anterior surface) report on microscopic margin involvement in $40-45 \%$ of DP specimens, which is markedly lower than in our series. ${ }^{32,34,35}$ However, the data are difficult to compare as the pathology examination protocols that were followed are hardly mentioned in these 
TABLE 4 Univariable and multivariable analysis of prognostic factors for overall survival following distal pancreatectomy for nonmetastatic ductal adenocarcinoma

\begin{tabular}{|c|c|c|c|c|}
\hline \multirow[t]{2}{*}{ Variables } & \multicolumn{2}{|l|}{ Univariable } & \multicolumn{2}{|l|}{ Multivariable } \\
\hline & HR $(95 \% \mathrm{CI})$ & $p$ value & HR $(95 \%$ CI $)$ & $p$ value \\
\hline Age, years & $1.01(0.98-1.03)$ & 0.82 & & \\
\hline Body mass index, $\mathrm{kg} / \mathrm{m}^{2}$ & $0.98(0.94-1.03)$ & 0.49 & & \\
\hline Male sex (vs. female) & $0.92(0.59-1.41)$ & 0.69 & & \\
\hline Period 2013-2020 (vs. 2004-2012) & $0.86(0.54-1.39)$ & 0.54 & & \\
\hline Red blood cell transfusion & $1.67(0.97-2.90)$ & 0.07 & - & - \\
\hline Extended resection (vs. standard) & $1.95(1.24-3.06)$ & 0.004 & $2.03(1.27-3.24)$ & 0.003 \\
\hline Severe complications & $1.18(0.72-1.96)$ & 0.51 & & \\
\hline Poor differentiation (vs. well/moderate) & $1.22(0.78-1.91)$ & 0.38 & & \\
\hline \multicolumn{5}{|l|}{ Tumor stage (vs. pT1) } \\
\hline pT2 & $1.46(0.64-3.33)$ & 0.37 & & \\
\hline pT3 & $1.84(0.83-4.08)$ & 0.13 & & \\
\hline \multicolumn{5}{|l|}{ Nodal stage (vs. pN0) } \\
\hline $\mathrm{pN} 1$ & $1.7(0.99-2.94)$ & 0.06 & - & - \\
\hline $\mathrm{pN} 2$ & $2.46(1.38-4.41)$ & 0.002 & - & - \\
\hline Lymph node yield & $1.01(0.99-1.03)$ & 0.46 & & \\
\hline Lymph node ratio (increase by 0.01 ) & $1.03(1.02-1.04)$ & 0.001 & $1.03(1.01-1.04)$ & 0.001 \\
\hline$R 1$ (vs. $R 0$ ) & $1.72(1.04-2.84)$ & 0.03 & - & - \\
\hline Positive transection margin & $1.34(0.71-2.55)$ & 0.37 & & \\
\hline Positive posterior margin & $1.1(0.71-1.69)$ & 0.66 & & \\
\hline Positive anterior surface & $2.49(1.57-3.94)$ & 0.001 & $2.03(1.26-3.26)$ & 0.004 \\
\hline$>1$ positive margin/surface & $1.8(1.1-2.96)$ & 0.02 & - & - \\
\hline Splenic vein invasion & $1.49(0.81-2.72)$ & 0.2 & & \\
\hline Lymphatic invasion & $1.44(0.91-2.28)$ & 0.12 & & \\
\hline Vascular invasion & $1.98(1.2-3.26)$ & 0.007 & - & - \\
\hline Perineural invasion & $2.86(1.24-6.59)$ & 0.014 & $3.9(1.6-9.4)$ & 0.003 \\
\hline Adjuvant chemotherapy & $0.54(0.35-0.83)$ & 0.006 & $0.41(0.25-0.66)$ & 0.001 \\
\hline
\end{tabular}

studies. Furthermore, since these were multicenter studies, the pathology examination methods may not have been uniform between the centers. In contrast, we have used a standardized pathology work-up (in period 2).

A key feature of the standardized grossing protocol used in period 2 is the emphasis on extensive tissue sampling. Indeed, this aspect is hardly ever mentioned in pathology protocols, including in those used for multicenter studies. Likewise, (inter-)national pathology guidelines do not provide any recommendations regarding the extent of sampling, and yet this is a decisive factor when it comes to the detection of $R 1$, i.e. microscopic margin/surface involvement. As this is, by definition, a microscopic finding and the invasive front of pancreatic cancer is notoriously difficult to identify on naked-eye inspection, more extensive tissue sampling from the tumor onto the adjacent specimen surface increases the likelihood of the detection of microscopic margin involvement. A positive correlation between the number of such tissue samples and the $R 1$ rate has been previously described for pancreatoduodenectomy specimens. ${ }^{8}$ In this study, the introduction of a more elaborate specimen examination procedure resulted in a significant increase in the median number of tissue blocks that were examined, i.e. 14 versus 9 in period $1(p<0.001)$. Given that the significant increase in the $R 1$ rate was observed concomitantly with the use of the new grossing protocol in 2015, we assume that the increased extent of sampling resulted in a higher detection rate of microscopic margin involvement in DP specimens.

One of the main objectives of this study was to examine the impact of $R 1$ resection on survival. Standard DP was associated with a significantly longer survival compared with extended DP regardless of the margin status of the former. $R 1$ was a significant prognostic factor for survival in the univariable analysis and in the subgroup analysis of patients from study period 2 . However, $R 1$ was not an independent predictor for survival, unlike factors such as the extent of surgery, anterior pancreatic surface status, 
lymph node ratio, perineural invasion, and adjuvant chemotherapy. In quantitative terms, the number of tumor cells in transit, either along the lymphatic or peripheral nerve system, is likely to be considerably higher than in one or two discrete foci with microscopic residual disease $(R 1)$. Hence, lymph node involvement and perineural invasion are probably stronger determinants of outcome, whereas $R 1$ may be only relevant for long-term outcome in node-negative patients without perineural invasion. However, because this patient group is very small, the establishment of a cohort sufficiently large to dissect out the prognostic impact of $R 1$ in these cases seems unrealistic.

To the best of our knowledge, this is the first study to report on the impact of the involvement of the anterior pancreatic surface on survival in patients undergoing DP for PDAC, however its prognostic role in pancreatic head cancer has been previously mentioned. ${ }^{36,37}$ Nonetheless, it did not correlate with either the development of recurrence or the site of recurrence, although a trend towards an increased risk of recurrence was seen in cases with involvement of the anterior surface. Our findings also demonstrate that involvement of the anterior pancreatic surface is associated with a higher $\mathrm{pN}$ stage, the presence of vascular invasion, involvement of more than one margin/surface, and poor tumor differentiation (electronic supplementary Table 5). Therefore, breaching the peritoneal lining of the pancreas by tumor cells may indicate a more aggressive tumor biology and/or a more advanced stage. Hence, specific reporting of involvement of the anterior surface rather than including it in the overall $R 1$ status without further specification may be relevant to the prediction of patient outcome. It should also be borne in mind that the anterior pancreatic surface is unaffected by the extent of surgery and that its involvement is rather determined by the location of the tumor. These findings indicate that neoadjuvant therapy may be considered when involvement of the anterior pancreatic surface and/or tumor ingrowth into adjacent organs is suggested on preoperative imaging. At the same time, it is worth mentioning that in seven of nine patients in this series who had received preoperative chemotherapy, DP resulted in an $R 1$ resection. This finding is in line with observations by others. ${ }^{38}$

The prognostic roles of lymph node ratio, perineural invasion, and adjuvant chemotherapy in pancreatic body/tail cancer have been reported previously. ${ }^{34,39,40}$ The dismal prognosis for patients undergoing extended DP for PDAC has been highlighted in our previous reports and in other studies. ${ }^{34,40-43}$ In this study, resection margins were positive in $82 \%$ of patients undergoing extended resection. Considering that all extended resection specimens that were examined according to the standardized protocol in period 2 showed $R 1$, we believe that $R 0$ can hardly be expected in this group. Nearly two-thirds of these patients had a positive posterior margin, while in over half, more than one margin/surface was involved (electronic supplementary Table 2). Furthermore, the rates of involvement of the anterior pancreatic surface and of more than one positive margin/surface were significantly higher compared with standard DP.

This study has several limitations. First and foremost, despite the prospectively collected database, this was an observational cohort study with its inherent weaknesses due to retrospectively defined parameters. Second, the findings of this study indicate that $R 1$ may have been underreported in period 1 , which, in turn, may have influenced our results regarding long-term oncologic outcomes. Third, only very few selected cases underwent open DP, thus there was no possibility to assess the potential influence of surgical approach on resection margin status. Lastly, due to the small number of patients who were treated with preoperative chemotherapy, the impact of the latter on resection margin status and prognosis could not be thoroughly evaluated.

\section{CONCLUSION}

A meticulous, standardized procedure for specimen grossing is key for the adequate assessment of margin status in DP specimens with PDAC. The systematic use of such a procedure shows that microscopic margin involvement occurs in the vast majority of cases. While the overall $\mathrm{R}$ status does not affect prognosis, involvement of the anterior pancreatic surface, extended DP, lymph node ratio, perineural invasion, and adjuvant chemotherapy are associated with survival.

Supplementary Information The online version contains supplementary material available at https://doi.org/10.1245/s10434021-10464-6.

ACKNOWLEDGMENT No grant support or other assistance was received to assist in the preparation of this manuscript.

FUNDING Open access funding provided by University of Oslo (incl Oslo University Hospital).

DISCLOSURES Mushegh A. Sahakyan, Caroline S. Verbeke, Tore Tholfsen, Dejan Ignjatovic, Dyre Kleive, Trond Buanes, Kristoffer Lassen, Bård I. Røsok, Knut Jørgen Labori, and Bjørn Edwin have no commercial interests or sources of financial or material support to declare.

OPEN ACCESS This article is licensed under a Creative Commons Attribution 4.0 International License, which permits use, sharing, adaptation, distribution and reproduction in any medium or format, as long as you give appropriate credit to the original author(s) and the source, provide a link to the Creative Commons licence, and indicate 
if changes were made. The images or other third party material in this article are included in the article's Creative Commons licence, unless indicated otherwise in a credit line to the material. If material is not included in the article's Creative Commons licence and your intended use is not permitted by statutory regulation or exceeds the permitted use, you will need to obtain permission directly from the copyright holder. To view a copy of this licence, visit http://creativecommons. org/licenses/by/4.0/.

\section{REFERENCES}

1. Tempero MA, Malafa MP, Chiorean EG et al. Pancreatic Adenocarcinoma, Version 1.2019. J Natl Compr Canc Netw. 2019;17(3):202-10

2. Johnston WC, Hoen HM, Cassera MA, et al. Total pancreatectomy for pancreatic ductal adenocarcinoma: review of the National Cancer Data Base. HPB (Oxford). 2016;18(1):21-8.

3. Konstantinidis IT, Warshaw AL, Allen JN, et al. Pancreatic ductal adenocarcinoma: is there a survival difference for R1 resections versus locally advanced unresectable tumors? What is a "true" R0 resection? Ann Surg. 2013;257(4):731-6.

4. Howard TJ, Krug JE, Yu J, et al. A margin-negative R0 resection accomplished with minimal postoperative complications is the surgeon's contribution to long-term survival in pancreatic cancer. J Gastrointest Surg. 2006;10(10):1338-45.

5. Chandrasegaram MD, Goldstein D, Simes J, et al. Meta-analysis of radical resection rates and margin assessment in pancreatic cancer. Br J Surg. 2015;102(12):1459-72.

6. Verbeke CS. Resection margins and R1 rates in pancreatic cancer-are we there yet? Histopathology. 2008;52(7):787-96.

7. Menon KV, Gomez D, Smith AM, Anthoney A, Verbeke CS. Impact of margin status on survival following pancreatoduodenectomy for cancer: the Leeds Pathology Protocol (LEEPP). HPB (Oxford). 2009;11(1):18-24.

8. Verbeke CS, Leitch D, Menon KV, McMahon MJ, Guillou PJ, Anthoney A. Redefining the R1 resection in pancreatic cancer. $\mathrm{Br}$ J Surg. 2006;93(10):1232-7.

9. Esposito I, Kleeff J, Bergmann F, et al. Most pancreatic cancer resections are R1 resections. Ann Surg Oncol. 2008;15(6):1651-60.

10. Gebauer F, Tachezy M, Vashist YK, et al. Resection margin clearance in pancreatic cancer after implementation of the Leeds Pathology Protocol (LEEPP): clinically relevant or just academic? World J Surg. 2015;39(2):493-9.

11. Chang DK, Johns AL, Merrett ND, et al. Margin clearance and outcome in resected pancreatic cancer. J Clinical Oncol. 2009;27(17):2855-62.

12. Osipov A, Nissen N, Rutgers J, et al. Redefining the positive margin in pancreatic cancer: impact on patterns of failure, longterm survival and adjuvant therapy. Ann Surg Oncol. 2017;24(12):3674-82.

13. Campbell F, Smith RA, Whelan P, et al. Classification of R1 resections for pancreatic cancer: the prognostic relevance of tumour involvement within $1 \mathrm{~mm}$ of a resection margin. Histopathology. 2009;55(3):277-83.

14. Lof S, Rajak R, Vissers F, et al. DIPLOMA approach for standardized pathology assessment of distal pancreatectomy specimens. J Vis Exp. 2020. https://doi.org/10.3791/60343.

15. Marangos IP, Buanes T, Rosok BI, et al. Laparoscopic resection of exocrine carcinoma in central and distal pancreas results in a high rate of radical resections and long postoperative survival. Surgery. 2012;151(5):717-23.

16. Sahakyan MA, Kim SC, Kleive D, et al. Laparoscopic distal pancreatectomy for pancreatic ductal adenocarcinoma: long-term oncologic outcomes after standard resection. Surgery. 2017;162(4):802-11.

17. Nordby T, Hugenschmidt H, Fagerland MW, Ikdahl T, Buanes T, Labori KJ. Follow-up after curative surgery for pancreatic ductal adenocarcinoma: asymptomatic recurrence is associated with improved survival. Eur J Surg Oncol. 2013;39(6):559-66.

18. Nagtegaal ID, Odze RD, Klimstra D, et al. The 2019 WHO classification of tumours of the digestive system. Histopathology. 2020;76(2):182-8.

19. von Elm E, Altman DG, Egger M, Pocock SJ, Gøtzsche PC, Vandenbroucke JP. The strengthening the reporting of observational studies in epidemiology (STROBE) statement: guidelines for reporting observational studies. Int $J$ Surg. 2014;12(12):1495-9.

20. Sahakyan MA, Haugvik SP, Rosok BI, et al. Can standardized pathology examination increase the lymph node yield following laparoscopic distal pancreatectomy for ductal adenocarcinoma? HPB (Oxford). 2018;20(2):175-81.

21. Verbeke C. Operative Specimen Handling and Evaluation of Resection Margins. In: SW Kim, H Yamaue, editors. Pancreatic cancer. With special focus on topical issues and surgical techniques. Part I. Pathology and tumor biology. Springer: Berlin; 2017. p. 67-87.

22. Campbell F, Verbeke C. Pathology of the pancreas: a practical approach. 2nd edn. Switzerland: Springer; 2021.

23. Hartwig W, Vollmer CM, Fingerhut A, et al. Extended pancreatectomy in pancreatic ductal adenocarcinoma: definition and consensus of the International Study Group for Pancreatic Surgery (ISGPS). Surgery. 2014;156(1):1-14.

24. Strasberg SM, Linehan DC, Hawkins WG. The accordion severity grading system of surgical complications. Ann Surg. 2009;250(2):177-86.

25. Bassi C, Marchegiani G, Dervenis C, et al. The 2016 update of the International Study Group (ISGPS) definition and grading of postoperative pancreatic fistula: 11 Years After. Surgery. 2016;161(3):584-91.

26. Wente MN, Veit JA, Bassi C, et al. Postpancreatectomy hemorrhage (PPH): an International Study Group of Pancreatic Surgery (ISGPS) definition. Surgery. 2007;142(1):20-5.

27. Brierley JD, Gospodarowicz MK, Wittekind C. TNM classification of malignant tumours. Eight. Oxford: Wiley Blackwell; 2017.

28. Amin MB, Edge SB, Greene FL, et al. AJCC cancer staging manual. Eight. New York: Springer; 2017.

29. Kawaguchi Y, Fuks D, Nomi T, Levard H, Gayet B. Laparoscopic distal pancreatectomy employing radical en bloc procedure for adenocarcinoma: technical details and outcomes. Surgery. 2015;157(6):1106-12.

30. Bauman MD, Becerra DG, Kilbane EM, et al. Laparoscopic distal pancreatectomy for pancreatic cancer is safe and effective. Surg Endosc. 2018;32(1):53-61.

31. Demir IE, Jager C, Schlitter AM, et al. R0 Versus R1 resection matters after pancreaticoduodenectomy, and less after distal or total pancreatectomy for pancreatic cancer. Ann Surg. 2018;268(6):1058-68.

32. Malleo G, Maggino L, Ferrone CR, et al. Number of examined lymph nodes and nodal status assessment in distal pancreatectomy for body/tail ductal adenocarcinoma. Ann Surg. 2019;270(6):1138-46.

33. Chen K, Tong Q, Yan JF, et al. Laparoscopic versus open distal pancreatectomy for pancreatic ductal adenocarcinoma: a singlecenter propensity score matching study. Updates Surg. 2020;72(2):387-97.

34. Korrel M, Lof S, van Hilst J, et al. Predictors for survival in an international cohort of patients undergoing distal pancreatectomy 
for pancreatic ductal adenocarcinoma. Ann Surg Oncol. 2021;28(2):1079-87.

35. de Rooij T, Tol JA, van Eijck $\mathrm{CH}$, et al. Outcomes of distal pancreatectomy for pancreatic ductal adenocarcinoma in the netherlands: a nationwide retrospective analysis. Ann Surg Oncol. 2016;23(2):585-91.

36. Japan Pancreas Society. Classification of pancreatic cancer. 2nd Edition (English). Kanehara, Tokyo; 2003.

37. Nagakawa T, Sanada H, Inagaki M, Sugama J, Ueno K, Konishi I, et al. Long-term survivors after resection of carcinoma of the head of the pancreas: significance of histologically curative resection. J Hepato Biliary Pancreat Surg. 2004;11:402-8.

38. Crippa S, Giannone F, Schiavo Lena M, et al. R status is a relevant prognostic factor for recurrence and survival after pancreatic head resection for ductal adenocarcinoma. Ann Surg Oncol. 2021;28(8):4602-12. https://doi.org/10.1245/s10434-02009467-6.

39. Kantor O, Bryan DS, Talamonti MS, et al. Laparoscopic distal pancreatectomy for cancer provides oncologic outcomes and overall survival identical to open distal pancreatectomy. $J$ Gastrointest Surg. 2017;21(10):1620-5.
40. Shin SH, Kim SC, Song KB, et al. Appraisal of laparoscopic distal pancreatectomy for left-sided pancreatic cancer: a large volume cohort study of 152 consecutive patients. PLOS ONE. 2016;11(9):e0163266.

41. Sahakyan MA, Kleive D, Kazaryan AM, et al. Extended laparoscopic distal pancreatectomy for adenocarcinoma in the body and tail of the pancreas: a single-center experience. Langenbecks Arch Surg. 2018;403(8):941-8.

42. Sahakyan MA, Kazaryan AM, Rawashdeh M, et al. Laparoscopic distal pancreatectomy for pancreatic ductal adenocarcinoma: results of a multicenter cohort study on 196 patients. Surg Endosc. 2016;30(8):3409-18.

43. Sulpice L, Farges O, Goutte N, et al. Laparoscopic distal pancreatectomy for pancreatic ductal adenocarcinoma: time for a randomized controlled trial? Results of an all-inclusive national observational study. Ann Surg. 2015;262(5):868-74.

Publisher's Note Springer Nature remains neutral with regard to jurisdictional claims in published maps and institutional affiliations. 